\title{
Monitoring the Impact of Heat Waves with Emergency Service Utilization Data in Los Angeles County
}

\author{
Emily Kajita*, Patricia Araki, Monica Luarca and Bessie Hwang \\ Los Angeles County Dept. of Public Health, Los Angeles, CA, USA
}

\section{Objective}

To assess current indicators for situational awareness during heat waves derived from electronic emergency department (ED) and 911 emergency dispatch call (EDC) center data.

\section{Introduction}

Los Angeles County's (LAC) early event detection system captures over $60 \%$ of total ED visits, as well as 800 to 1,000 emergency dispatch calls from Los Angeles City Fire (LACF) daily. Both ED visits and EDC calls are classified into syndrome categories, and then analyzed for aberrations in count and spatial distribution. During periods of high temperatures, a heat report is generated and sent to stakeholders upon request. We describe how syndromic surveillance serves as an important near real-time, population-based instrument for measuring the impact of heat waves on emergency service utilization in LAC.

\section{Methods}

Daily electronic ED registration data, EDC calls, and high temperatures from Palmdale, California were queried from January 1, 2010 to August 26, 2012 and aggregated into Centers for Disease Control (CDC) weeks. A custom "heat exposure" category was created by searching ED chief complaints for key terms such as "Heat stroke," "hyperthermia," "overheat," and relevant ICD9 diagnosis codes. Similarly, EDC calls were classified if related to "heat exposure." Pearson correlation tests were used to determine correlation between total ED visits, heat-related ED visits, heat-related EDC calls, and daily maximum temperatures.

\section{Results}

Thus far 2012 has exceeded counts cumulative to August 26th for the past two years in the number of heat-related ED visits, heat-related EDC calls, and hot days (Table 1). In particular, the number of 105 degree-and-up days this year has already doubled what was observed all year during 2010 and 2011.

Age groups were similarly distributed in total ED visits, heat-related ED visits and EDC calls, with a 18 to 44 year old majority (37\%, $37 \%$, and $42 \%$ respectively), followed by 45 to 64 year olds $(23 \%$, $21 \%, 23 \%$ ). Total ED visits did not increase during summer months, and were therefore not found to be correlated to temperature $(\rho=-$ $0.06, p=0.46)$ or heat-related EDC calls $(\rho=0.07, p=0.4)$. Heat-related ED visits however, were positively correlated with both EDC calls $(\rho=0.85, p<0.001)$ and temperatures $(\rho=0.59, p<0.001)$. Heat-related EDC calls were also correlated with temperature $(\rho=0.56, p<0.001)$.

\section{Conclusions}

Due to small numbers of heat-related visits relative to total ED visits, any effects that increased temperatures may have on total ED visits are undetectable. Total ED volume should therefore not be used as an indicator for measuring the impact of heat on LAC's population.
Filtering chief complaints to obtain heat-specific ED visits, however, enables patterns of increase to emerge which correlate with higher temperatures and heat-related emergency dispatch calls. About $35 \%$ of the week to week variation in heat-related ED visits, and 32\% of the week to week variation in heat-related EDC calls can be explained by week to week variations in temperature. That heat-related visits were similarly distributed in age as all visitors suggests that heat does not disproportionately affect children and the elderly any more than the other acute health conditions that bring visitors to the ED. Syndromic analysis of ED data and EDC can provide baselines for health conditions such as hyperthermia that are otherwise difficult to obtain.

\begin{tabular}{|c|c|c|c|}
\hline & 2010 to $8 / 26$ (year end total) & $\begin{array}{c}2011 \text { to } 8 / 26 \\
\text { (year end total) }\end{array}$ & 2012 to $8 / 26$ \\
\hline Heat-related ED visits & $214(319)$ & $195(304)$ & 246 \\
\hline Heat-related 911 calls & $102(169)$ & $73(128)$ & 163 \\
\hline Number of days $>80 \mathrm{~F}$ & $102(148)$ & $99(152)$ & 123 \\
\hline Number of days $>90 \mathrm{~F}$ & $72(100)$ & $67(96)$ & 87 \\
\hline Number of days $>100 \mathrm{~F}$ & $18(23)$ & $14(17)$ & 27 \\
\hline Number of days $>105 \mathrm{~F}$ & $3(3)$ & $4(4)$ & 7 \\
\hline
\end{tabular}

Table 1. Number of heat-related ED visits, EDC calls, and days exceeding temperatures to $8 / 26$ and to the year's end.

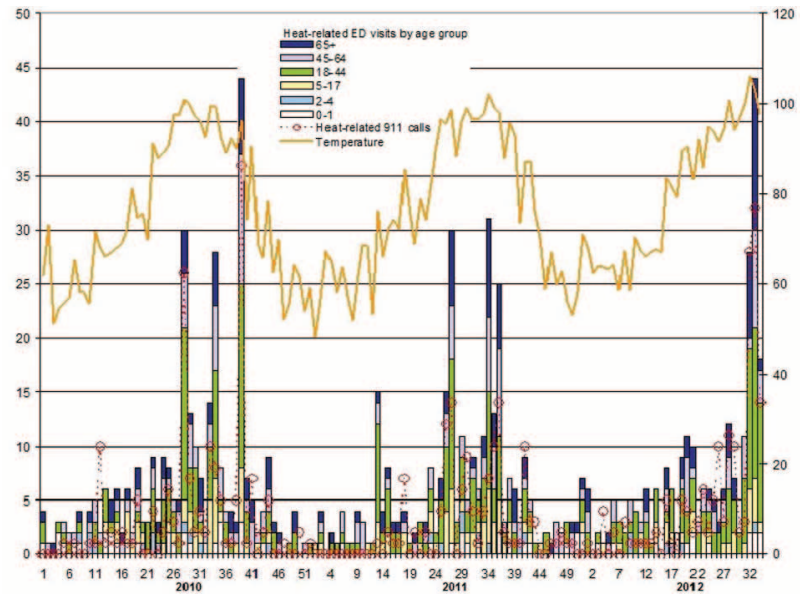

Figure 1. Weekly heat-related ED visits and heat-related EDC calls (left axis); and 7-day averaged maximum daily temperatures in Palmdale, California (right axis). Heat-related ED visits are stratified by age group.

\section{Keywords}

Emergency department; Dispatch; Heat; Situational awareness

\section{References}

Weather underground. 2012. 27 Aug. 2012. The Weather Channel Companies. $<$ http://www.wunderground.com/ $>$

\section{*Emily Kajita}

E-mail: ekajita@ph.lacounty.gov 